\title{
Automated Emergency Rescue Alert System
}

\author{
Muralidhar Reddy Pattikonda, Sreenivasula Reddy Pottipati, Srinivasulu Senduru
}

\begin{abstract}
In the present days for the patients to go out, by leaving their designated care area is one of the important risks because of accidents or so. In the proposed system we have attempted to allow medical staff or the care taker to monitor and to reach the landmark using the automatic alert systems. If an accident occurs, immediately the working staffs will get alerts with location and point that minimizing response times. In the project, we use an Accident identification method by detecting the accident through Vibration sensor which is connected to the android application to detect its location, this will save the life of the people by sending the ambulance on time even without receiving any call alerts from the public. We also deploy an android based women safety so that we can be rescued before the happening of any physical harassment. Age old people can also send any request medical emergency support.
\end{abstract}

\section{INTRODUCTION}

Nowadays it is normal that accident happens daily and will be taking to hospital where they care for all patients. But it is unsure about they are alive or not. So it is important to track those data's using an automated system. Hence an automated machine will reflects with location and time when it happens time. It is important to track the exact location where the incident happened. Therefore, we are concentrating on long term caring systems to offer better quality and also reasonable cost. Hence this will make the old age peoples and the person who is disabled to go anywhere independently without any fear or problem [16,17]. Such facilities have to be implemented using the recent techniques with proper ideas and management. The rescue of people who met accident became difficult and to detect location also make more problem. It is difficult to make call or send message to ambulance when major accident occurs[18]. The Emergency alert message will send to register number when any accident occurred. The location will be shared to medical staff and family then required action will be taken on time even without receiving any alerts from public. We also make use of android base safety so that we can rescue woman before happening of physical harassment.

\section{RELATED WORK}

The paper deals with illustration and evaluation of continuous monitoring of eyes and also the pose of head for alerting the driver of the vehicle. Many present proposals depended on visual identification of non alertness driving models that depend further more on head nodding angles or eye closure to detect driver distraction level or drowsiness.

Revised Version Manuscript Received on 10 September, 2019.

Muralidhar Reddy Pattikonda, UG Student, Dept. of CSE,

Sreenivasula Reddy Pottipati, UG Student, Dept. of CSE, Sathyabama University, Chennai, Tamilnadu, India.

Dr. Srinivasulu Senduru, Assistant professor, Dept. of IT, Sathyabama University, Chennai, Tamilnadu, India. and so it is easy for medical staffs to reach the location on Sathyabama University, Chennai, Tamilnadu, India.

This approached scheme using visual characteristics such are eye index (EI), pupil activity (PA), and head position (HP) to take out data of the vehicle driver non alertness. EI is detecting whether eyes are fully opened or half closed, or fully closed through the ratio of the height of the eye and height of the pupil. Pupil activity is used to measure the ratio of difference between the centers of the eye for different phase of time. The head position is used to evaluate the driver's movement of head by different parameters of metrics such as tilting the head, nodding the head and shaking the heads from the normal position of driving. Hence this will be tracked based on the driver's attention while driving. Hence many researchers have developed many algorithms in order to overcome the issue and even now the research is going on the same topic. SVM (Support Vector Machines) have been proposed to classify the driver's activity using the conversion of frames from a video. Hence the classification of layers will help them to assess and to deliver better output of alert. Hence the evaluation of proposed model shows that the system performs better in terms of classification and delivers better output at the end. Also they have provided less error and false alarm rate is less with true alarm rate is high. Therefore the output shows better recognition rate in the proposed work [1].

The most important point everyone discuss daily is the accidents happening on road because of Driver fatigue. This happens because of continuous driving or driving without taking any rest will become sleepiness that causes accidents, and hence it is important to give awareness about this. From recent past years a large number of techniques were examined on driver drowsiness detection. This paper deals with these techniques survey. By observing the driving pattern these techniques will detect the driver drowsiness. The driver drowsiness state is hypothesized through abnormalities in driving pattern. In this purpose various measures like behavioral, vehicular, physiological, and subjective have used. The technique proposed will not need help of drivers it is fully based on the behavioral parameters. So it is fully based on the head movement parameters from among different behavioral measures paper [2].

Fall accident detection and recue system is a high achievement in the medical industry .It is based on environmental monitoring based or wearable sensor based system. But now in the era of modern world smart phone is acting as a sensor. The angels obtained by digital compass and waveform order of tri-axial accelerometer on smart phone used as inputs while fall detection. When the accident happens at that point the location of that incident happened will be transferred through GPS via $3 \mathrm{G}$ system. This will help 
them to get an better treatment of immediate basis through the proposed approach [3].

The paper is checking of the attentiveness condition of a driver which has challenge to examination in safety and management of transportation. In this approach we deal with a real time robust embedded program for maintaining and observe the dropping of driver attentiveness in driving conditions through during day and night. The eye closure proportion of eye is used to find the wariness level of driver in driving conditions. The face detection is detected using the features like Haar and using a Kalman filter is tracked. The detection of eyes during day time is recognized through principal component analysis and during night using features of block local-binary-pattern. Finally, with support vector machines the eye state is identified weather open or closed. In-plane rotation of the face of the driver is been detected using of affine transformation and off-plane is through using of perspective transformation. Illumination variation is carried with bi-histogram equalization. The algorithm is cross-validated with using brain signals and it implement on single-board computer which has Intel Atom processor with 1.66-GHz, $\times 86$ architecture, $1 \mathrm{~GB}$ random access memory, and XP operating system which is in Windows-embedded. The system is robust under driving conditions Paper [4].

In this paper, an indoor position system is proposed in emergency situations for situation awareness. A system that provide positioning information which uses inertial sensors without GNSS coverage in environments. We provide entire system with indoor position and mapping web portal which are deployed in smart phone application and given positioning data access paper [5].

In this paper a WBAN method is implemented to address hardware, software implementations and designs of wireless protocol. Using WABN system an healthcare applications is deployed which deals with sensor nodes to obtain physiological data through using WBAN medical bands. Through reduction of interference there is an increase in previous nodes and hence by using the medical gateway wireless boards transformation of collected data to a scheduled position using multi-hopping methods. Here the facilities acquirable in health centers and medical professions access patient's physiological signals. paper[6].

Women's security is a basic issue in this day and age and it's particularly required for each person to act over such an issue. This paper portrays a "GPS and GSM based women security system" that gives the mix of GPS gadget and specific programming to follow the vehicle's area just as furnish alarms and messages with a crisis catch trigger. Presently days because of as of late happened cases, for example, assault by drivers or partners, theft and so forth., representative security, particularly women worker security has turned into the first need of the organizations. System utilizes the Global Positioning System innovation to discover the area of vehicle. The data of vehicle position given by the gadget can be seen on Google maps utilizing Internet or particular programming. The IT Companies are anticipating the security issue and requires a system that will effectively assess the issue of women representatives' security working in night shifts. This paper centers on the proposed model to overcome the issue of security concerns of women group using GPS and GSM based system. One of recent discussed topic is the security of a woman and how important is to protect them or to make them independent to roam anywhere at any point of time is the main focus in terms of security.

\section{METHODOLOGY}

We add on Accident identification by detecting the accident through Vibration sensor which is connected to the android application to detect its location, this will save the life of the people by sending the ambulance on time even without receiving any call alerts from the public. We also deploy an android based women safety so that we can be rescued before the happening of any physical harassment Age old people can also send any request for medical emergency support. In this system we need single application to send alert notification in emergency situation.

\section{Architecture Diagram}

The diagram refers the flow of project process and mechanism. The main object is to share the location of emergency situation.

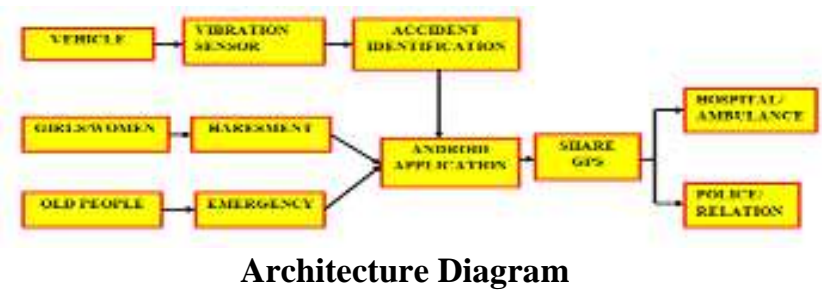

\section{EMBEDDED HARDWARE FABRICATION}

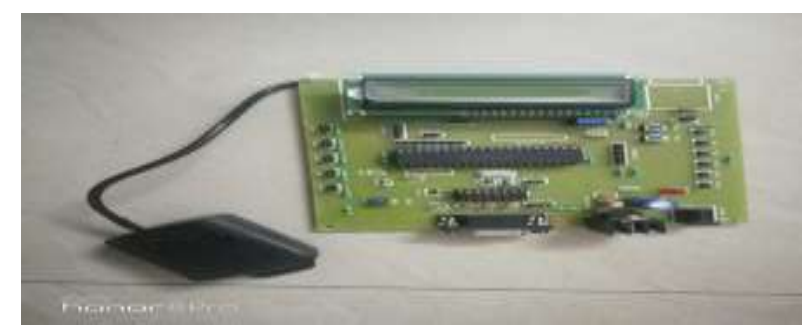

\section{Embedded Hardware Kit}

In this project we use IOT kit which will be connected to smart phone through cable. The embedded kit should be placed in the vehicle dash board which is ahead to vehicle driver. The main component is vibration sensor which activates when an accident occurs. The LCD displays the activation of kit. Different types of resistors are used which provides stability by providing correct current flow and signal strengths and voltage for the kit. We use microchip which is used for the collection of data and it also sends the information to the right location or destination. We use HDMI port which transfers data to the required -device. The HDMI transfers the data like audio type, images and video type. We use the HDMI cable to connect the device to the HDMI jack port. GSM modem is used for communication. Power input can be done through power jack.

\section{MODULES}

1. Android user

2. Android mobility

\section{Published By:}

Blue Eyes Intelligence Engineering

\& Sciences Publication 
3. Embedded hardware fabrication

4. Women's safety

5. Emergency Alert

\section{Android User}

User has to register their details to access the application. They have to register three types of registration first they have to register their name, guardian number, police number, Doctor number Alternate number to transfer the message. Second we have to register some types of unlock system. Finally, some static questions will be asked by application for security.

\section{Android Mobility}

In this module we create an application for secured authentication. In all the Android mobiles customer has to install the mobile client application. Hence it will help one for the user friendly activity. Hence the process starts with registration and it is important to create the user login forum using texts in the android phones. It also contain start, edit, register button which leads to further process.

\section{Embedded Hardware Fabrication}

This module is about the hardware connection on hardware kit we use vibration sensor, heart beat sensor and PIC board are connected together. Heart beat sensor will identify the old people health condition, if old people are in emergency situation the system will automatically send alert to the ambulance. Vibration sensor which activates when an accident occurs.

\section{Women's Safety}

In this module, unlocking system will make only in mobile. Firstly, we should register and then we will insert and submit details. We can change details if it required. We can set a pattern of tilting positions. When an emergency situation occurs the user has to move the mobile in tilting position. So that system can send an alert message which contain GPS location to registered mobile number, parents and police. Thus, the user can save and supported.

\section{Emergency Alert}

In this module, we identify the accident and send notification to the ambulance. Vibration sensor will identify the accident and send it to the server.

Regulation Survey of Accidents.

Table: Road accident statistics.

\begin{tabular}{|c|c|c|c|}
\hline$\gamma=a x$ & $\begin{array}{l}\text { Aceldanto } \\
\text { per yeur }\end{array}$ & $\begin{array}{l}\text { Numbar of } \\
\text { perxonx } \\
\text { Winnd }\end{array}$ & $\begin{array}{l}\text { Number of } \\
\text { perxonx } \\
\text { lnjured }\end{array}$ \\
\hline 2017 & 1986:177 & 1.9757 .3 & 10.1594 \\
\hline 2014 & $48: 301$ & $13: 1072$ & $4: 347=$ \\
\hline 2012 & 501424 & 146134 & 500280 \\
\hline 2016 & dsanc:s. & 1 × & 00.4625 \\
\hline $201 /$ & $404: 111$ & $147: 314$ & $470: 170$ \\
\hline
\end{tabular}

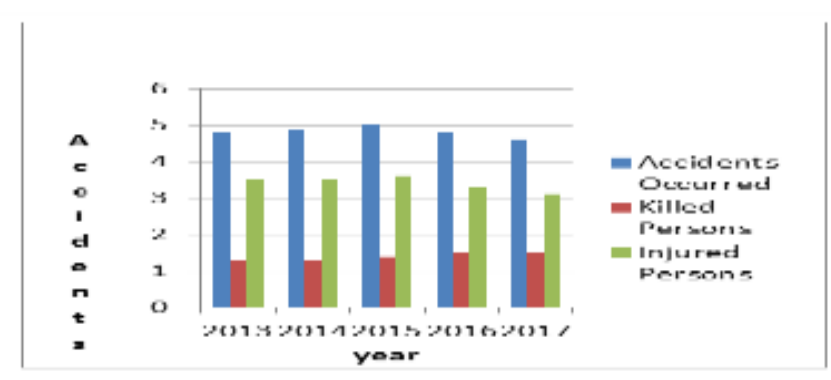

\section{GPS(Global Positioning System) role of GPS}

The GPS system is used to send the data of the location to the required person. Here the main aim of the project is send location of victim to the rescue team and family members. The GPS is a system here the latitude coordinates .

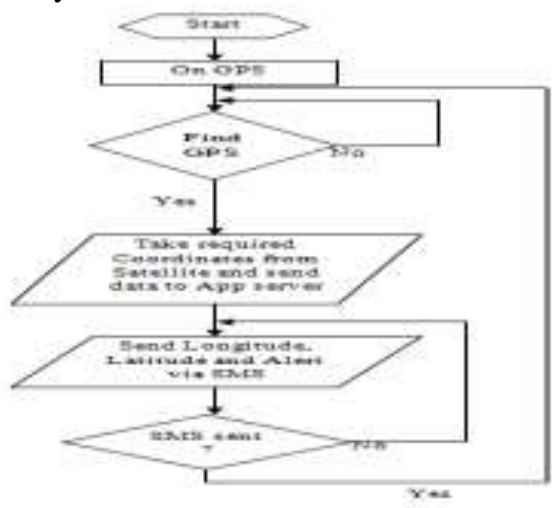

GPS is defined as global positioning system which is used to navigate the data about the point of time and the position at any circumstances to monitor the user.

It consists of three sections

I. Space Section.

II. Control System Section.

III. User Section.

\section{RESULT}

In this woman safety system, we will have an android application which is deployed in mobile. The application is created in android studio by using java code. A server which is building on other side to connect the mobile. Firstly, the application contains three buttons EDIT, REGISTER. The user has to register at first by clicking register button.
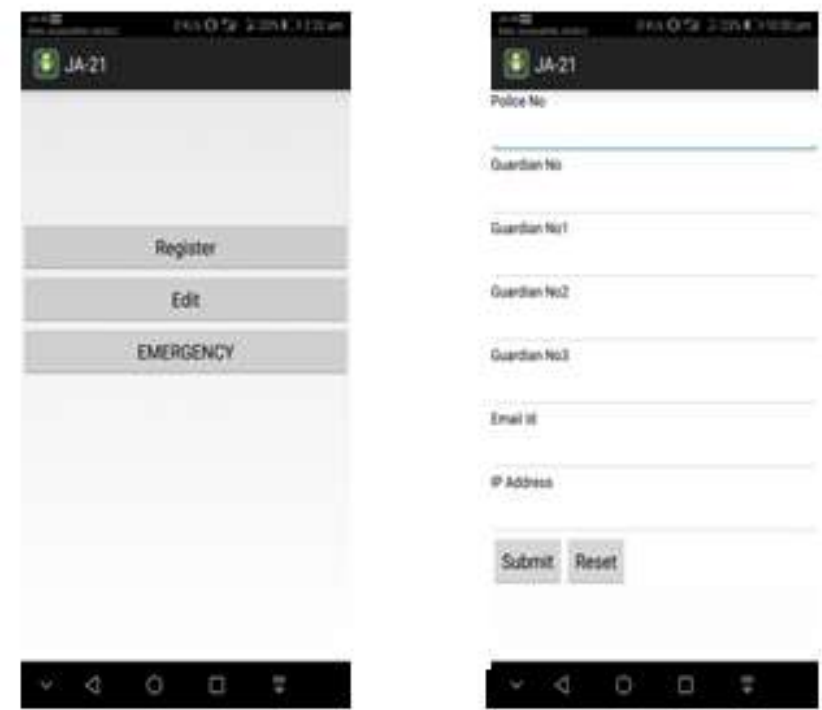

The user has to provide details like phone number, guardian number, E-mail id, IP Address and a password has to set which resemble tilting pattern. We can set various tilting patterns according to user. They can change pattern through edit button. 


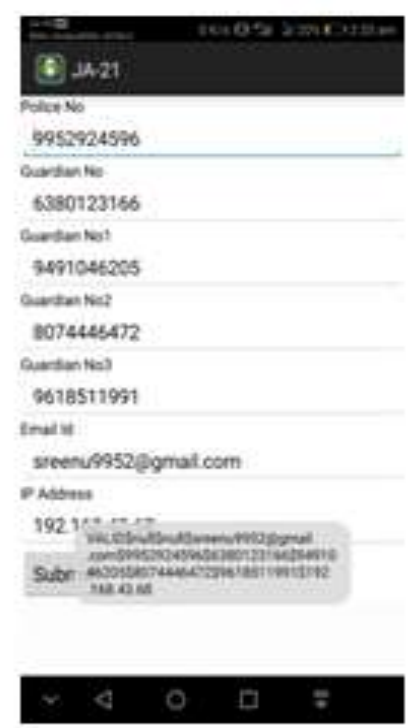

After registration of user details in the application, the details will be stored in server database. After that, if any harassment or incident is going to happen means then user has to tilt the phone in that specific pattern. Then application will active and send an alert message to registered phone numbers. The process will start by sending request to server then server will accept the request and send response message to register number. Thus the required action can be taken to save the user.

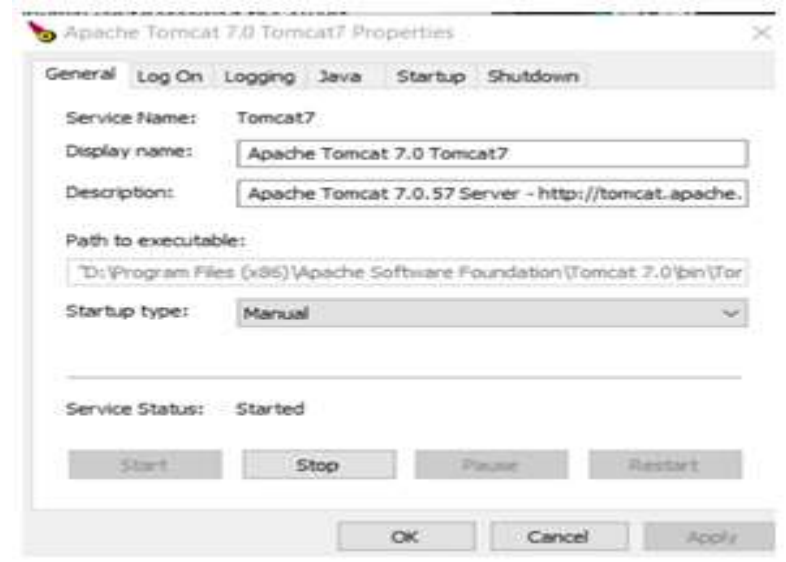

\section{CONCLUSION}

Today accidents and women harassments are increasing day by day. More number of security systems was came but crimes are increasing rapidly. Our proposed system will solve those problems by using android application. When an accident occurred one place, vibration sensor will gather the information and send notification to the police and medical staff that accident occurred. So, that person can get saved. If any women affected by arrestments, she has to move the mobile in tilting position. So that system can send an alert message which contain GPS location to registered mobile number, parents and police. Thus, the user can save and supported. Finally the patient tracking if any problem occurred notification will send to the medical staff.

\section{REFERENCES}

1. Ralph Oyini Mbouna, Seong G. Kong, Myung-Geun Chun, "Visual Analysis of Eye State and Head Pose for
Driver Alertness Monitoring", IEEE journal on intelligent transportation systems, vol. 14, no. 3, september 2013

2. Ajay Mittal, Kanika Kumar, Sarina Dhamija , M. Kaur," Head movement-based driver drowsiness detection: A review of state-of-art techniques", Vol. 5 (3) , 2014, 4245-4249

3. L.-J. Kau and C.-S. Chen, "A smart phone-based pocket fall accident detection, positioning, and rescue system," IEEE journal of biomedical and health informatics, pp. 44-56, 2015.

4. Anirban Dasgupta, Anjith George, S. L. Happy, and Aurobinda Routray, "A Vision-Based System for Monitoring the Loss of Attention in Automotive Drivers", Vol 4 no 7, July 2015

5. L. Berbakov, B. Pavkovic and S. Vrane, "Smart Indoor Positioning System for Situation Awareness in Emergency Situations," in 26th International Workshop on Database and Expert Systems Applications (DEXA), 2015, pp. 139-143.

6. M. R. Yuce, "Implementation of wireless body area networks for healthcare systems," Sensors and Actuators A: Physical, vol. 162, no. 1, pp. 116-129, 2010.

7. J. C. Williams, 2012 Sleep in America poll: Transportation workers' sleep, 2011.

8. Y. Dong, Z. Hu, K. Uchimura, N. Murayama, "Driver inattention monitoring system for intelligent vehicles: A review", IEEE Trans. Transp. Syst., vol. 12, no. 2, pp. 596-614, Jun. 2011.

9. V. Gumster, The Complete Guide to Blender Graphic: Computer Modeling and Animation, USA, FL, Boca Raton:CRC, 2012.

10. Xuesong Wang, Chuan $\mathrm{Xu}$, "Driver drowsiness detection based on non-intrusive metrics considering individual specifics", Accident Analysis and Prevention, 2013.

11. Jos Vicente et al., "Drowsiness detection using heart rate variability", Medical and biological engineering and computing, pp. 1-11, 2016.

12. R. Libby, "A simple method for reliable footstep detection on embedded sensor platforms", 2008.

13. G. Milette, A. Stroud, Professional Android Sensor Programming, John Wiley and Sons, Inc., 2012

14. H. Ghafghazi, A. Elmougy, H. T. Mouftah and C. Adams, "Location-Aware Authorization Scheme for Emergency Response," IEEE Access, pp. 4590-4608, 2016.

15. R. Zhang, F. Hoflinger and L. Reindl, "Inertial sensor based indoor localization and monitoring system for emergency responders," IEEE Sensors Journal, pp. 838-848, 2013

16. Monisha, D.G.,Monisha, M.,Pavithra, G.,Subhashini, R.,"Women safety device and application-FEMME ", Indian Journal of Science and Technology, Vol 9(10), DOI: 10.17485/ijst/2016/v9i10/88898, March 2016.

17. Dr.R.Subhashini and Milani.V, "IMPLEMENTING GEOGRAPHICAL INFORMATION SYSTEM TO PROVIDE EVIDENT SUPPORRT FOR CRIME ANALYSIS", International Conference on Intelligent Computing, Communication \& Convergence(ICCC-2014), Bhubaneswar, Odisha on 27th-28th December, 2014.

18. Sanjanna, Y. Bevish Jinila (2015), "An Approach on Automated Rescue System with Intelligent Traffic Lights for Emergency Services", International Conference on Innovations in Information, Embedded and Communication Systems (ICIIECS 2015), pp. 1-5, IEEE. 